\title{
Robotic-assisted thoracoscopic surgery (RATS) lobectomy
}

\author{
Giye Choe, Bernard Park \\ Thoracic Surgery Service, Department of Surgery, Memorial Sloan Kettering Cancer Center, New York, USA \\ Correspondence to: Bernard Park. Thoracic Surgery Service, Department of Surgery, Memorial Sloan Kettering Cancer Center, New York, USA. \\ Email: parkb@mskcc.org.
}

Submitted Jan 30, 2019. Accepted for publication Feb 20, 2019.

doi: $10.21037 /$ acs.2019.02.07

View this article at: http://dx.doi.org/10.21037/acs.2019.02.07

\section{Clinical vignette}

A 56-year-old female former smoker presented with a 5 -cm right upper lobe (RUL) mass invading the chest wall discovered during work up for shoulder pain (Figures 1,2). The extent of disease was investigated with a whole body FDG PET/CT, which revealed uptake in the mass, hilar and mediastinal lymph nodes, but no distant locations. Brain MRI was negative. Pre-treatment mediastinal staging with endobronchial ultrasound (EBUS) was negative for nodal disease, and the patient was clinically staged as T3N0M0. Given the locally advanced nature of her disease and her pain, induction chemoradiation was offered. She underwent treatment with concurrent chemoradiation using cisplatin/pemetrexed and 50 Gy of radiation. Post treatment PET/CT demonstrated decreasing avidity and decrease in the size of the mass to $3.5 \mathrm{~cm}$. The patient's pain largely resolved. She was otherwise healthy and her preoperative pulmonary function tests (PFT) showed $\mathrm{FEV}_{1}$ of $2.4 \mathrm{~L}$ (113\% expected) and DLCO $16.9 \mathrm{~mL} / \mathrm{mmHg} / \mathrm{min}(80 \%$ expected). The patient was offered robotic lobectomy with concomitant chest wall resection.

\section{Surgical techniques}

\section{Preparation}

After induction of general anesthesia and lung isolation with a double lumen endotracheal tube, the patient is positioned in a lateral decubitus position with the operating table maximally flexed. The robotic surgical cart is positioned on the ipsilateral side of the table (Xi system) or the table is turned so that the cart is above the patient's head ( $\mathrm{Si}$ system).

\section{Exposition}

We employ a robotic-assisted minimally invasive surgical (MIS) approach utilizing a total of five incisionsfour robotic and one non-robotic-with selective $\mathrm{CO}_{2}$ insufflation. All hilar dissection and isolation of the structures are performed with robotic instrumentation, while certain aspects of the procedure (parenchymal stapling, chest wall division) are achieved using non-robotic MIS instrumentation.

\section{Operation}

\section{Docking and port placement}

The initial and most critical port fashioned is in the $7^{\text {th }}$ or $8^{\text {th }}$ intercostal space (ICS) just posterior to the posterior axillary line for the camera. An $8-\mathrm{mm}$ robotic port is introduced, and the camera is introduced into the pleural space to rule out advanced disease and to place the other ports under direct vision. A 30-degree scope angled down is preferred, however zero degree is feasible as well. Two posterior $8-\mathrm{mm}$ ports are next placed-the first in the $8^{\text {th }}$ or $9^{\text {th }}$ ICS just posterior to the tip of the scapula. The fenestrated bipolar forceps are utilized at this port during hilar dissection, and a conventional endovascular stapler or robotic stapler (Xi system only) can be introduced here for division of the hilar structures. The second posterior port is placed in the $5^{\text {th }}$ or $6^{\text {th }}$ ICS at the border of the paraspinal muscle at the level of the junction of the superior segment and the basilar segments of the lower lobe. For the $\mathrm{Xi}$ system a tip-up fenestrated grasper is used for retraction of the lung tissue, whereas for the Si system the $5-\mathrm{mm}$ thoracic grasper is utilized. The final $8-\mathrm{mm}$ robotic port is placed through a $3-\mathrm{cm}$ anterior incision in the $5^{\text {th }}$ ICS in the 


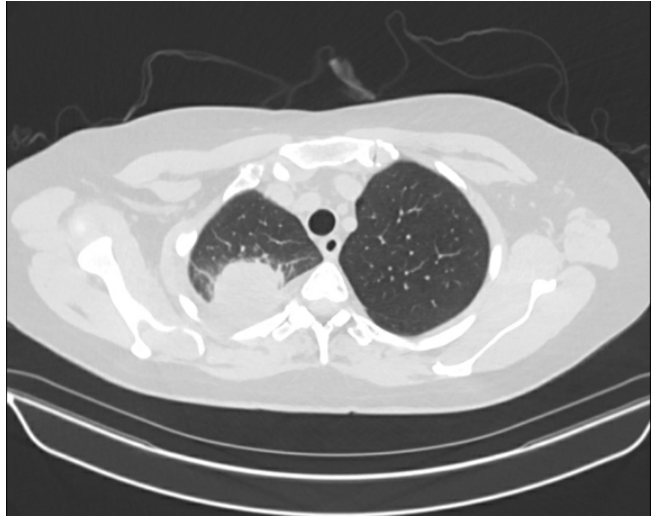

Figure 1 Axial image of right upper lobe tumor.

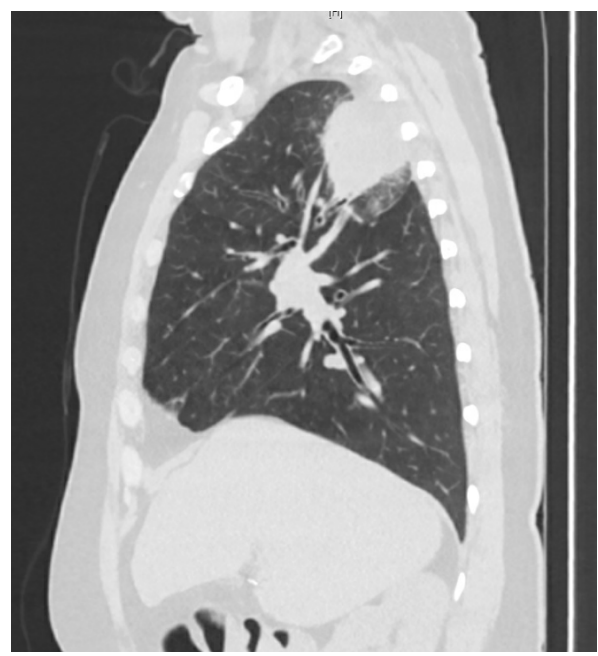

Figure 2 Sagittal image of right upper lobe tumor.

mid-axillary line, and a monopolar spatula is utilized there. A $5 \mathrm{~mm}$ non-robotic port is placed between the camera and the first posterior port for the bedside assistant. $\mathrm{CO}_{2}$ insufflation to $8-10 \mathrm{mmHg}$ is employed only in cases where greater intrathoracic space is desired (obese patient with high riding diaphragm or severe emphysema and delayed absorptive atelectasis).

\section{Posterior hilar and mediastinal dissection}

In all cases of anatomic pulmonary resection, it is the authors' preference to begin with posterior hilar and mediastinal dissection. The lower lobe is retracted superiorly, and the inferior pulmonary ligament is divided with electrocautery. The inferior ligament and periesophageal nodes are removed. The lung is then retracted anteriorly, and the posterior pleura is divided at its interface with the lung parenchyma up to the superior extent of the hilum near the azygos vein. The hilar lymph nodes are individually removed. In the right chest, this includes the interlobar "sump" nodes between the RUL and the bronchus intermedius. This facilitates isolation and division of the RUL bronchus from either the posterior or anterior approach. A subcarinal lymph node dissection is performed.

\section{RUL bilar dissection}

Following the posterior hilar and mediastinal dissection, it is our practice to perform an anterior-to-posterior approach without dissection in the fissure. The lung is retracted laterally in order to place the superior hilar vessels on tension. The mediastinal pleura over the superior pulmonary vein is incised in order to delineate the entire extent of the upper lobe vein. The superior extent is where the vein meets the truncus arteriosus, while the inferior extent is at the takeoff of the middle lobe vein. Care must be taken to identify the middle lobe vein inferiorly and to preserve it. There are typically hilar lymph nodes in these two areas, and they should be excised or mobilized away from the vein. Prior to isolation and division of the upper lobe vein, the ongoing pulmonary artery should be identified and avoided. Once isolated, the upper lobe vein is divided with an endovascular stapler introduced through the posterior inferior port exposing the basilar pulmonary artery and the truncus arteriosus. Division of the pleural reflection is continued superiorly around the hilum until the RUL bronchus is reached.

Next, the truncus is mobilized from the surrounding hilar lymph nodes inferiorly and the upper lobe bronchus posteriorly and divided. At this juncture, the peribronchial lymph nodes and any remaining sump nodes that have not been previously excised should be removed completely. This maneuver will result in complete mobilization of the posterior ascending branch of the upper lobe bronchus and will clearly delineate the location of the posterior ascending artery branch. These two remaining hilar structures may then be divided in whichever order is practically easiest. On occasion where the posterior ascending branch arises more proximal on the main pulmonary artery, it is necessary to divide this branch first. The bronchus is typically stapled with a $4.8-\mathrm{mm}$ stapler or may be divided sharply and sewn closed with 3-0 or 4-0 absorbable suture. Lastly, the horizontal fissure is completed with multiple fires of a linear stapler. Following removal of the lobectomy specimen the 
right paratracheal lymph node dissection is performed. All tissue from the trachea to the superior vena cava the azygos vein to the thoracic inlet and down to the level of the pericardial reflection is removed.

In the case of our clinical vignette, we completed the lobectomy first and then performed the chest wall resection of ribs 2 and 3 en bloc with the tumor. After lysing several flimsy adhesions and performing a limited extrapleural dissection to mobilize the uninvolved lung the intercostal muscle above and below the involved ribs was divided. The anterior margins were circumferentially dissected out, and a thoracoscopic rib cutter was used through the anterior incision to divide the ribs laterally. Then the ribs were reflected forward and mobilized away from the overlying musculature. Eventually, the rib heads were disarticulated off the transverse processes using cautery and blunt dissection. In this case ability of the robot to exert considerable force was useful because it allowed for superior counter traction required to disarticulate the rib heads.

\section{Completion of the procedure}

After completion of the lobectomy and en bloc chest wall resection, a polypropylene specimen bag was introduced through the anterior port site to remove the specimen from the chest. The robotic instruments are removed, and the surgical cart is undocked. A single $28 \mathrm{~F}$ chest tube is left in place through the camera port incision, the remaining lung re-expanded, and the incisions are closed in standard fashion.

\section{Comments}

We first described our technique of robotic assistance for video-assisted thoracic surgical (VATS) lobectomy in 2006 (1). We reported on 30 cases of peripheral tumors without evidence of regional metastases that were done with robotic assistance and demonstrated that this was a feasible method with perioperative outcomes comparable to conventional VATS lobectomy ( $12 \%$ conversion to open rate, no perioperative mortalities, morbidity rate of $26 \%$, and a median hospital stay of 4.5 days). Since then, the use of robotic assisted VATS has steadily increased and in 2012 an international multi-institutional retrospective review of 325 cases of early stage non-small cell lung cancer was published focusing on long term oncologic outcomes (2). We found that perioperative outcomes were similar to conventional VATS and open approaches $(8 \%$ conversion to open rate, morbidity rate of $25.2 \%$, one in- hospital death $(0.03 \%)$ and median hospital stay of 5 days). Long term oncologic outcomes were comparable (overall 5 -year survival of $80 \%, 91 \%$ for pathologic stage IA, $88 \%$ for stage IB, $49 \%$ for stage II disease. Three-year survival of $43 \%$ for IIIA disease). Finally, we published a study reviewing our experience of robotic assisted lobectomy, conventional VATS lobectomy and open lobectomy in 2016 (3). This study demonstrated that while most of the outcomes for the three techniques were similar, there was a statistically significant difference in the median number of lymph node stations harvested (5 for robotic $v s .3$ for VATS vs. 4 for open; $\mathrm{P}<0.001)$. This suggests that the robotic platform allows for a more thorough lymph node harvesting compared to other approaches. There was a statistically significant difference between the 5-year disease-free survival $(72.7 \%$ in robotic vs. $65.5 \%$ in VATS, $\mathrm{P}=0.047)$ but multivariate analysis found that surgical approach was not independently associated with shorter overall and diseasefree survival. These studies show that robotic assisted lobectomy is not only a feasible and safe surgery but may have oncologic benefits as well.

\section{Advantages}

The high definition, magnified binocular (3D) visual system combined with the instrumentation with seven degrees of freedom allow for more precise dissection of soft tissue compared with the traditional VATS approach. This confers a significant advantage when performing complete nodal dissection. Reports suggesting a higher rate of nodal upstaging compared to thoracoscopic surgery support this $(3,4)$. These same technological advantages may also facilitate more difficult cases, such as patients who have undergone induction therapy, obese patients with limited intrathoracic space, and patients with locally advanced disease. Moreover, despite the lack of haptic feedback the ergonomic design of the console, motion scaling, and lack of fulcrum effect decrease musculoskeletal fatigue in the surgeon and allows for consistent performance even throughout longer, difficult cases and perhaps leading to lower need for conversion (5). Lastly, for treatment of primary lung cancer there are increasing reports of longterm oncologic outcomes commensurate with traditional open approaches $(2,6)$.

\section{Caveats}

Mastering the numerous advantages of the robotic platform 
requires a steep learning curve, both for the surgeon and the institution. Success requires a multidisciplinary team effort between surgeon, nursing, anesthesiology and the operating room team. A capable, experienced bedside assistant is critical. The initial and ongoing costs of the platform is high and cost-reduction and alternative platforms are needed.

\section{Acknowledgements}

Funding: This work was funded, in part, by the National Institutes of Health/National Cancer Institute Cancer Support Grant P30 CA008748.

\section{Footnote}

Conflicts of Interest: Dr. Bernard Park has served as a clinical proctor and received honorarium from Intuitive Surgical. Dr. Giye Choe has no conflicts of interest to declare.

\section{References}

1. Park BJ, Flores RM, Rusch VW. Robotic assistance for

Cite this article as: Choe G, Park B. Robotic-assisted thoracoscopic surgery (RATS) lobectomy. Ann Cardiothorac Surg 2019;8(2):296-299. doi: 10.21037/acs.2019.02.07 video-assisted thoracic surgical lobectomy: technique and initial results. J Thorac Cardiovasc Surg 2006;131:54-9.

2. Park BJ, Melfi F, Mussi A, et al. Robotic lobectomy for non-small cell lung cancer (NSCLC): long-term oncologic results. J Thorac Cardiovasc Surg 2012;143:383-9.

3. Yang HX, Woo KM, Sima CS, et al. Long-term Survival Based on the Surgical Approach to Lobectomy For Clinical Stage I Nonsmall Cell Lung Cancer: Comparison of Robotic, Video-assisted Thoracic Surgery, and Thoracotomy Lobectomy. Ann Surg 2017;265:431-7.

4. Wilson JL, Louie BE, Cerfolio RJ, et al. The prevalence of nodal upstaging during robotic lung resection in early stage non-small cell lung cancer. Ann Thorac Surg 2014;97:1901-6; discussion 1906-7.

5. Reddy RM, Gorrepati ML, Oh DS, et al. Robotic-Assisted Versus Thoracoscopic Lobectomy Outcomes From High-Volume Thoracic Surgeons. Ann Thorac Surg 2018;106:902-8.

6. Cerfolio RJ, Ghanim AF, Dylewski M, et al. The longterm survival of robotic lobectomy for non-small cell lung cancer: A multi-institutional study. J Thorac Cardiovasc Surg 2018;155:778-6. 